\title{
Editorial: Virtual Reality, Augmented Reality and Video Games for Addressing the Impact of COVID-19 on Mental Health
}

\author{
Federica Pallavicini ${ }^{1 *}$, Irene Alice Chicchi Giglioli ${ }^{2}$, Gerard Jounghyun Kim ${ }^{3}$, \\ Mariano Alcañiz ${ }^{2}$ and Albert Rizzo ${ }^{4}$
}

${ }^{1}$ Department of Human Sciences for Education "Riccardo Massa", University of Milano Bicocca, Milan, Italy, ${ }^{2}$ Instituto de Investigación e Innovación en Bioingeniería (i3B), Universitat Politécnica de Valencia, Valencia, Spain, ${ }^{3}$ Korea University, Seoul, South Korea, ${ }^{4}$ Institute for Creative Technologies, University of Southern California, Los Angeles, CA, United States

Keywords: virtual reality, augmented reality, video games, COVID-19, mental health

Editorial on the Research Topic

Virtual Reality, Augmented Reality and Video Games for Addressing the Impact of COVID-19 on Mental Health

Since 2020, the worldwide coronavirus 2019 (COVID-19) pandemic has been reported to have increased the frequency of adverse mental health and stress-related conditions, including, depression, anxiety disorders, PTSD, and substance use (Pfefferbaum and North, 2020; Xiong et al., 2020; Niles et al., 2021). Changes in lifestyle behaviors, social isolation, boredom, financial security, and general uncertainty appear to have had a negative impact on the mental health of populations globally, with potential long-lasting psychological and physical consequences (Brooks et al., 2020; Moreno et al., 2020; Salari et al., 2020).

Virtual reality (VR), augmented reality (AR), and video games (VGs) appear as some of the most appealing technological interventions for addressing the impact of COVID-19 on mental health (Granic et al., 2014; Imperatori et al., 2020; Riva et al., 2020; Singh et al., 2020; Viana and De Lira, 2020; Woolliscroft, 2020; Barr and Copeland-Stewart, 2021; Rizzo et al., 2021 in press). Therefore, we brought together within this Research Topic contributions from researchers investigating theoretical, empirical, experimental, and case studies of VR, AR, and VGs for addressing the impact of COVID19 on mental health.

This article was submitted to
Virtual Reality in Medicine,
a section of the journal Frontiers in Virtual Reality

Received: 02 June 2021 Accepted: 21 June 2021 Published: 14 July 2021

Citation: Pallavicini F, Chicchi Giglioli IA, Kim GJ, Alcañiz M and Rizzo A (2021) Editorial: Virtual Reality, Augmented Reality and Video Games for Addressing the Impact of COVID-19 on Mental Health.

Front. Virtual Real. 2:719358 doi: 10.3389/frvir.2021.719358

\section{OPPORTUNITIES AND BARRIERS OF VR FOR SUPPORTING MENTAL HEALTH IN THE ERA OF COVID-19}

Pimental et al. discuss the promise of VR to improve mental health by connecting communities to clinical and social support systems. The authors identify socioeconomic barriers to widespread VR use, delineated across five dimensions (i.e., sociocultural, content, affordability, supply chain, and equitable design). Solutions that can ensure this technology can be equitably accessed via existing, and future infrastructure changes are discussed.

Sampaio et al. explore how therapists are using telepsychology before vs. during the COVID-19 pandemic. An online survey on 768 mental health professionals showed that before COVID-19, most therapists only saw their patients in person. However, during the COVID-19 pandemic, nearly all therapists used a wide range of telecommunication technologies to communicate with their patients, 
including texting, telephones, video conferences, and even VR. Although this study reported that VR is rarely used by the therapists surveyed, according to the authors, in the future, therapists and patients in physically different locations will be able to "meet" in a shared computer-generated world designed for therapy sessions, potentially including group sessions.

Stradford et al. provide methods for launching a clinical trial during the COVID-19 pandemic. They carried out a pilot study on five healthy female participants aged between 51 and 76 years that uses an in-lab brain and body training program to promote brain health in mid-to-late life older adults. Based on the findings of this pilot study, the authors suggest that continued VR clinical trial research during the COVID-19 pandemic is achievable and can be safely resumed if specific safety protocols are in place to mitigate the risk of exposure and spread of COVID-19.

\section{COMMERCIAL-OFF-THE SHELVES VIDEO GAMES AND AD HOC VIRTUAL REALITY CONTENTS FOR PSYCHOLOGICAL SUPPORT DURING THE COVID-19 PANDEMIC}

Lewis et al. investigate whether participation in the famous socializing and life simulation game "Animal Crossing: New Horizons" was related to emotional outcomes associated with pandemics (e.g., loneliness and anxiety). Results of an online survey conducted on 1,053 participants showed that increased gaming and related activities predicted higher anxiety and increased loneliness. However, increased

\section{REFERENCES}

Barr, M., and Copeland-Stewart, A. (2021). Playing Video Games during the COVID-19 Pandemic and Effects on Players' Well-Being. Games Cult. 155541202110170. doi: $10.1177 / 15554120211017036$

Brooks, S. K., Webster, R. K., Smith, L. E., Woodland, L., Wessely, S., Greenberg, N., et al. (2020). The Psychological Impact of Quarantine and How to Reduce it: Rapid Review of the Evidence. The Lancet 395 (10227), 912-920. doi:10.1016/S0140-6736(20)30460-8

Granic, I., Lobel, A., Engels, R. C. M. E., and Engels, R. C. M. E. (2014). The Benefits of Playing Video Games. Am. Psychol. 69 (1), 66-78. doi:10.1037/a0034857

Imperatori, C., Dakanalis, A., Farina, B., Pallavicini, F., Colmegna, F., Mantovani, F., et al. (2020). Global Storm of Stress-Related Psychopathological Symptoms: A Brief Overview on the Usefulness of Virtual Reality in Facing the Mental Health Impact of COVID-19. Cyberpsychology, Behav. Soc. Networking 23 (11), 782-788. doi:10.1089/cyber.2020.0339

Moreno, C., Wykes, T., Galderisi, S., Nordentoft, M., Crossley, N., Jones, N., et al. (2020). How Mental Health Care Should Change as a Consequence of the COVID-19 Pandemic. The Lancet Psychiatry 7 (9), 813-824. doi:10.1016/ S2215-0366(20)30307-2

Niles, J. K., Gudin, J., Radcliff, J., and Kaufman, H. W. (2021). The Opioid Epidemic within the COVID-19 Pandemic: Drug Testing in 2020. Popul. Health Manag. 24 (S1), S-43. doi:10.1089/pop.2020.0230

Pfefferbaum, B., and North, C. S. (2020). Mental Health and the COVID-19 Pandemic. N. Engl. J. Med. 383 (6), 510-512. doi:10.1056/nejmp2008017

Riva, G., Mantovani, F., and Wiederhold, B. K. (2020). Positive Technology and COVID-19. Cyberpsychology, Behav. Soc. Networking 23 (9), 581-587. doi:10.1089/cyber.2020.29194.gri

Rizzo, A., Hartholt, A., and Mozgai, S. (2021). From Combat to COVID19-Managing the Impact of Trauma Using Virtual Reality. J. Tech. Hum. Serv. doi:10.1080/15228835.2021.1915931 visits to another island were associated with lower levels of loneliness. According to the authors, players may be utilizing gaming as a coping mechanism for anxiety.

Pallavicini et al. detail MIND-VR's design and evaluation protocol, a virtual reality-based psychoeducational experience on stress and anxiety developed following a user-centered design approach. The virtual experience will be tested on a sample of Italian hospital healthcare personnel involved in the COVID-19 pandemic emergency and is available free of charge, both in Italian and English, on the project website (https://mindvr.com/).

\section{CONCLUSION}

The papers published on this Research Topic provide an overview of the applications of VR, AR, and VGs for supporting mental health during the COVID-19 pandemic, giving relevant insights for future developments in their adoption for remote psychological support. We believe that these technologies can soon revolutionize the way to support mental health, creating solutions and programs low-cost and easily accessible to millions of people worldwide.

\section{AUTHOR CONTRIBUTIONS}

All authors listed have made a substantial and intellectual contribution to the work and approved for publication.

Salari, N., Hosseinian-Far, A., Jalali, R., Vaisi-Raygani, A., Rasoulpoor, S., Mohammadi, M., et al. (2020). Prevalence of Stress, Anxiety, Depression Among the General Population during the COVID-19 Pandemic: A Systematic Review and Meta-Analysis. Glob. Health 16 (1), 57. doi:10.1186/ s12992-020-00589-w

Singh, R. P., Javaid, M., Kataria, R., Tyagi, M., Haleem, A., and Suman, R. (2020). Significant Applications of Virtual Reality for COVID-19 Pandemic. Diabetes Metab. Syndr. Clin. Res. Rev. 14 (4), 661-664. doi:10.1016/ j.dsx.2020.05.011

Viana, R. B., and De Lira, C. A. B. (2020). Exergames as Coping Strategies for Anxiety Disorders during the COVID-19 Quarantine Period. Games Health J. 9 (3), 147-149. doi:10.1089/g4h.2020.0060

Woolliscroft, J. O. (2020). Innovation in Response to the COVID-19 Pandemic Crisis. Acad. Med. 95 (8), 1140-1142. doi:10.1097/ACM.0000000000003402

Xiong, J., Lipsitz, O., Nasri, F., Lui, L. M. W., Gill, H., Phan, L., et al. (2020). Impact of COVID-19 Pandemic on Mental Health in the General Population: A Systematic Review. J. Affective Disord. 277, 55-64. doi:10.1016/ j.jad.2020.08.001

Conflict of Interest: The authors declare that the research was conducted in the absence of any commercial or financial relationships that could be construed as a potential conflict of interest.

Copyright (c) 2021 Pallavicini, Chicchi Giglioli, Kim, Alcañiz and Rizzo. This is an open-access article distributed under the terms of the Creative Commons Attribution License (CC BY). The use, distribution or reproduction in other forums is permitted, provided the original author(s) and the copyright owner(s) are credited and that the original publication in this journal is cited, in accordance with accepted academic practice. No use, distribution or reproduction is permitted which does not comply with these terms. 